\section{Ambivalent proaction following common label pretraining}

\author{
M. E. MARSHALL \\ Carleton University, Ottawa 1, Canada
}

Twenty Ss were assigned to each of three experimental treatments, the transfer effects of which were assessed in a subsequent discriminative motor task. One group learned different verbal responses to each of six test-task stimuli (24-point random shapes), while Ss in a second group learned two such responses to clusters of three of the same stimuli. A co trol group learned two verbal responses to clusters of different stimuli. Reliable differences were obtained between the treatment groups in terms of total test-task errors and two component error measures. An apparent interaction was observed between speed of original learning, treatment, and component error type.

When Ss learn identical verbal labels to ilusters of two or more visual stimuli within a training list. it is expected that performance will be retarded on subsequent test tasks involving stimulus recognition, discrimination learning, or the association of new instrumental responses to each training stimulus. Such relevant common label (RCL) training has been hypothesized to promote interference through the acquisition of identical verbal response-produced cues or common "attentional" responses to clusters of test list stimuli. The results of several studies employing recognition test tasks (e.g., Ellis, Bessemer, Devine, \& Trafton, 1962; Ellis \& Muller, 1964) indicate that RCL training does produce interference with respect to a control treatment (RO) that requires $\mathrm{Ss}$ merely to observe test list stimuli during training. With respect to the same RO control, Ss who learn a different label for each test-task stimulus (RDL training) show facilitation on the recognition task, at least under conditions of high stimulus complexity and intermediate numbers of training stimulus exposures. Such results have been interpreted as consistent with the hypotheses of acquired equivalence and distinctiveness of cues, though Ellis \& Muller (1964) caution that transfer effects inferred from differing performances following labeling and observation treatments may include nonspecific effects of practice in making overt responses to stimuli as well as the specific effects of stimulus differentiation.

Relatively little is known about the proactive effects of $\mathrm{RCL}$ training on test tasks that require new discriminative instrumental responses to be learned to each training stimulus. Using such a task, both Ellis \& Muller (1964) and del Castillo \& Ellis (1968) have demonstrated significant facilitation following RDL training, yet in the latter study, an RCL treatment group performed as well as the RO control. In another study (Marshall, 1968b), the consequences of
RCL and RJI training were compared with those of an irrelevant (ICL) control treatment involving verbal training of the CL type on stimuli different from those of the transfer task. Not unexpectedly, Ss made fewer errors following RDL training than after either ICL or RCL training. Disconcertingly, however, the RCL treatment produced significantly fewer errors than the ICL control, a finding at odds with the theory that learning common labels serves solely to increase effective stimulus similarity. These data suggested that the $R C L$ treatment may simultaneously produce interference and facilitation.

The experiment reported here was designed to explore this suggestion using a component error analysis of the performance of an RCL training group and its appropriate ICL control. Also of interest was the verification of the order of performance of Groups RDL and RCL obtained in the earlier study. The experimental design and the treatment of results were based on the following argument: If, during RCL training, a single response $(\mathrm{Ra})$ is learned to one cluster of, say, three stimuli ( $1, S 2$, and S3) and if a discriminably different response $(\mathrm{Rb})$ is learned to another cluster of stimuli (S4, S5, and S6) in a six-item list, one can simultaneously make the case for:

(1) Acquired equivalence of cues within cluster (WC)-The effective similarity between stimuli within the same cluster should increase by the addition through conditioning of a common response-produced stimulus element (and/or through the encoding of common stimulus features within cluster). The behavioral outcome of this process should be an increase in the number of generalized responises within cluster (WC errors, e.g., R3 elicited by $\mathrm{S} 1$ or R6 elicited by S5) relative to an appropriate $\mathrm{ICL}$ control. between cluster (BC)-The effective similarity between stimuli of different
(2) Acquired distinctiveness of cues clusters should decrease by virtue of the addition through conditioning of a discriminably different response-produced stimulus element (and/or through the encoding of discriminably different stimulus features between cluster). The behavioral outcome of this process should be a decrease in the number of generalized responses between stimuli of different clusters ( $B C$ errors, e.g., R5 elicited by $\mathrm{S} 1$ or $\mathrm{R} 1$ elicited by $\mathrm{S} 4$ ) relative to the $\mathrm{ICL}$ control. Indeed, in terms of BC errors. the consequences of $\mathrm{RCL}$ training should resemble the consequences of $R D L$ (distinctiveness) training.

\section{METHOD}

The Ss were 60 students enrolled in Introductory Psychology. They were assigned to one of three training conditions: Ss in Group RDL learned the responses cow, tax, men, big, fur, and joy to the six stimuli (24-point random shapes) of the subsequent test task; Ss in Group RCL learned the response cow to one cluster of three of the stimuli and the response tax to the remaining three shapes; Ss in Group ICL learned these same two responses to two clusters of three different stimuli equated for complexity. Within Groups RCL and ICL, stimulus-response pairings were arranged such that over Ss, each stimulus appeared in a cluster with each other stimulus an equal number of times. Acquisition for all Ss proceeded by the paced paired-associates anticipation method, using a 4-sec stimulus exposure. Correction was administered orally by $E$ as soon as $S$ made a response or, if no response was made during the $4-\mathrm{sec}$ exposure interval, during the interexposure interval (ca. $0.6 \mathrm{sec}$ ). All Ss were brought to a criterion of three successive blocks of six correct anticipations.

The 20 trials of the paced paired-associates motor task were also administered by the anticipation-correction method using a lever-pulling apparatus and a visual correction procedure described elsewhere (Marshall, 1968a). All Ss were informed that on this task a different motor response was to be learned to each of the six presented shapes.

\section{RESULTS \\ Pretraining}

The mean number of trials to criterion on the training task were $22.9,19.9$, and 16.1 for Groups RDL, RCL, and ICL, respectively. When the distributions of trials to criterion were compared using the Mann-Whitney U test, neither the difference between Groups RDL and RCL $\left(\mathrm{U}=158, \mathrm{p}>.05, \mathrm{n}_{1}=\mathrm{n}_{2}=20\right)$ nor that between Groups RCL and ICL $(U=189$. $p>.05, n_{1}=n_{2}=20$ ) proved statistically significant. 
Total Transfer Errors

The mean number of errors per $S$ per trial on the test task were $1.45,1.90$, and 2.41 for Groups RDL, RCL, and ICL, respectively. An analysis of variance applied to the data for groups over 20 test trials yielded significant main effects for groups $(p<.01, F=6.66$, $d f=2,57)$ and for trials $(p<.01, F=50.68$, $\mathrm{df}=19,1083)$. The interaction of groups with trials was not statistically reliable $(p>.20)$. Individual $t$ tests based on the mean square for Error $b$ of the analysis of variance revealed significant differences between Groups RCL and ICL $(p<.05$, $t=1.94, \mathrm{df}=57)$ and between Groups $\mathrm{RCL}$ and $\mathrm{RDL}(\mathrm{p}<.05, \mathrm{t}=1.71, \mathrm{df}=57)$.

\section{WC and BC Transfer Errors}

On the basis of chance, Ss were expected to make more $\mathrm{BC}$ errors than WC errors. Hence, statistical analyses were performed for $B C$ and WC errors separately. For this purpose, arbitrary stimulus clusters were defined for Groups RDL and ICL in terms of the real clusters that had been arranged for Ss of Group RCL during the training task. For example, when Stimuli 1, 2, 3 formed the first and Stimuli 4, 5, 6 formed the second cluster during verbal training for $S 1$ in Group RCL, Stimuli 1, 2, 3, and $4,5,6$ were designated as the two stimulus clusters during the test task for $S 1$ in Group ICL and S 1 in Group RDL as well.

The analysis of variance applied to the WC error measure yielded significant effects associated with groups $(F=4.19$, $\mathrm{p}<.05, \mathrm{df}=2,57)$ and trials $(\mathrm{F}=17.53$, $\mathrm{p}<.01, \mathrm{df}=19,1083$ ), while the Trials by Groups interaction was not statistically reliable. The order of group means

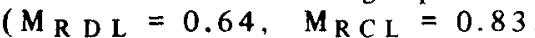
$M_{I C L}=1.02$ ) was the same as the order for total errors.

The analysis applied to $\mathrm{BC}$ errors also revealed significant groups ( $F=4.01$, $\mathrm{p}<.05, \mathrm{df}=2,57)$ and trials $(\mathrm{F}=22.63$, $\mathrm{p}<.01, \mathrm{df}=19,1083$ ) effects. The Groups by Trials interaction was not significant. The order of group means $\left(\mathrm{M}_{\mathrm{RDL}}=0.86\right.$, $\mathrm{M}_{\mathrm{RCL}}=1.05, \mathrm{M}_{\mathrm{ICL}}=1.35$ ) was the same as those based on WC and total error measures.

An examination of Table 1, showing the WC and $B C$ error data for the six fastest and six slowest training task learners, reveals an apparent interaction between acquisition speed, error type, and treatments. Statistical comparisons between the distributions of interest were performed using the Mann-Whitney U test.

For fast learners, none of the apparent differences between groups within error type proved reliable, although a $\mathrm{U}$ of 8 for the difference between RDLWC and ICLWC distributions is associated with
Table 1

Mean Number of WC and BC Errors Made During the Transfer Task

\begin{tabular}{|c|c|c|c|}
\hline \multirow{2}{*}{$\begin{array}{l}\text { Error } \\
\text { Type } \\
\end{array}$} & \multirow{2}{*}{$\begin{array}{l}\text { Training } \\
\text { Treatment }\end{array}$} & \multicolumn{2}{|c|}{$\begin{array}{l}\text { Speed of Training } \\
\text { Task A cquisition }\end{array}$} \\
\hline & & Fast & Slow \\
\hline WC & $\begin{array}{l}\text { RDL } \\
\text { RCL } \\
\text { ICL }\end{array}$ & $\begin{array}{l}0.51 \\
0.90 \\
0.96\end{array}$ & $\begin{array}{l}0.65 \\
0.66 \\
1.25\end{array}$ \\
\hline $\mathrm{BC}$ & $\begin{array}{l}\text { RDL } \\
\text { RCL } \\
\text { ICL } \\
\end{array}$ & $\begin{array}{l}0.95 \\
0.75 \\
1.28 \\
\end{array}$ & $\begin{array}{l}1.00 \\
1.02 \\
1.38 \\
\end{array}$ \\
\hline
\end{tabular}

$p<.06\left(n_{1}=n_{2}=6\right)$.

For slow learners, however, the $U$ of 6 for the RCLWC-ICLWC distributions is significant $\left(p<.032, n_{1}=n_{2}=6\right)$, as is the $\mathrm{U}$ of 5 for distributions RDLWC and ICL $W C\left(p<.021, n_{1}=n_{2}=6\right)$. No group difference is significant for the $\mathrm{BC}$ error measure.

\section{DISCUSSION}

Since the distribution of trials to training-task criterion for Group RCL did not differ reliably from the distributions for Groups RDL and ICL, the proactive effect of training-task difficulty was assumed equivalent across treatment groups.

RDL training resulted in fewer errors on the test task than either $R C L$ or ICL training; $\mathrm{RCL}$ training resulted in fewer errors than did ICL training. These results confirm earlier evidence (Marshall, 1968b) that the number of verbal training responses and relevancy of training are significant sources of transfer in the $\mathrm{AB}-\mathrm{AC}$ predifferentiation paradigm.

In the introduction, it was argued that the RCL treatment should induce acquired equivalence within stimulus cluster alone, and that between stimulus clusters, the same treatment should induce acquired distinctiveness. The behavioral outcomes predicted by these arguments are (1) Group RCL should make as many if not more errors than Group ICL on the test task in terms of WC errors, but (2) in terms of the $\mathrm{BC}$ error measure, Group RCL should make fewer errors than Group ICL-as few, in fact, as Group RDL.

Contrary to expectation, separate analyses of $\mathrm{BC}$ and $\mathrm{WC}$ errors revealed no relations that were not obvious in the analysis of total errors. The rank order of performance for the three experimental groups was constant over total, WC, and BC errors.

The data viewed differentially for fast and slow Ss, however, suggest an interaction between training acquisition speed, training treatment, and error type. These data indicate that slow learners benefit as much from RCL training as from RDL training, such that slow RCL and $\mathrm{RDL}$ Ss share a common superiority to slow learners in the ICL group in terms of both WC and BC error measures. Similarly, fast learners in Groups RCL and RDL made lewer $\mathrm{BC}$ errors than did their $\mathrm{ICL}$ counterparts, supporting the assertion that RCL training establishes the conditions for acquired distinctiveness between the stimuli of different clusters.

However, on the WC error measure, fast learners in Group RCL made more errors than their counterparts in Group RDL, and almost as many errors as fast $\mathrm{Ss}$ in Group ICL. These relations are in the direction predicted by the arguments of the introduction, suggesting that RCL training may indeed establish the conditions for acquired equivalence within stimulus cluster, but for fast learners alone.

The reliability of the interaction between training acquisition speed, training treatment, and error type must be determined in experiments specifically designed to explore the speed of acquisition variable, for the interaction received little statistical support in the present study. It is suggested, however. that differential effects of RCL training for fast and slow learners might well rest on the use of differential tactics for the solution of the training task. RCL Ss probably all first seek common features of stimuli within cluster. If these common features are found, $S$ should reach a fast solution and enter the test task with an S-R associative complex that conforms to the acquired equivalence paradigm. If common cues between stimuli with in cluster cannot be found, however, these Ss will be forced to resort to the time-consuming tactic of associating each stimulus individually with the common response demanded of a given cluster. These conditions should lead to slower acquisition during training, and should also result in Ss' entry to the test task with an S-R associative complex functionally resembling the acquired distinctiveness paradigm.

\section{REFERENCES}

DEL CASTILLO, D. M., \& ELLIS, H. C. The role of response-produced cues in paired-associates transfer as a function of intralist stimulus similarity. Psychonomic Science, 1968, 10. 197-198.

ELLIS, H. C., BESSEMER, D. W., DEVINE, J. V., \& TRAFTON, C. L. Recognition of random tactual shapes following predifferentiation training. Perceptual \& Motor Skills, 1962, 10, 99-102.

ELLIS, H. C., \& MULLER, D. G. Transfer of perceptual learning following stimulus predifferentiation. Journal of Experimental Psychology, 1964, 68, 388-395.

MARSHALL, M. E. Some effects of amount of verbal pretraining and availability of verbal labels upon performance in a discriminative motor task. Perceptual \& Motor Skills, 1968a, 26, 27-39.

MARSHALL, M. E. Common label pretraining: Interference and/or facilitation? Psychonomic Science, $1968 \mathrm{~b}, 13,313-314$. 